\title{
Shi-Style Cervical Mobilizations Versus Massage for Cervical Vertigo: A Multicenter, Randomized, Controlled Clinical Trial
}

\author{
Min Yao, PhD, Zhan-ying Tang, PhD, ${ }^{1}$ Xue-jun Cui, PhD, Yue-li Sun, PhD, \\ Xiu-lan Ye, PhD, ${ }^{2}$ Ping Wang, PhD, ${ }^{3}$ Wei-hong Zhong, MD, Rui-chun Zhang, MD, \\ Hui-ying Li, MD, ${ }^{6}$ Zhi-jun Hu, PhD, ${ }^{2}$ Wei-min Wang, MD, ${ }^{3}$ Wei-ping Qiao, MD, \\ Jun Li, MD, ${ }^{2}$ Yang Gao, MD, ${ }^{2}$ Qi Shi, BS, and Yong-jun Wang, PhD ${ }^{1}$
}

\begin{abstract}
Objectives: Large sample and high-quality evidence to evaluate the preliminary safety of the mobilizations and massage for cervical vertigo are not yet available. Thus, the present study aimed to investigate the comparative effectiveness and preliminary safety of Shi-style cervical mobilizations (SCM) compared with traditional massage (TM) in cervical vertigo patients.

Design: A prospective, multicenter, open-label, randomized, controlled clinical trial with a 1:1 allocation ratio.

Settings: Five academic medical centers.

Subjects: A total of 360 adult patients with a diagnosis of cervical vertigo.

Interventions: The patients were randomly allocated to either an SCM $(n=180)$ or TM $(n=180)$ group. The patients were treated during six sessions over 2 weeks. The primary outcome was the Dizziness Handicap Inventory (DHI) total scale score, and secondary outcomes included the DHI subscales, Chinese version of the Short-Form 36 Health Survey (CSF-36), and adverse events (AEs). Outcomes were assessed in the short term at 2 weeks, 1 month, and 3 months, and in the intermediate term at 6 months after randomization.

Results: Significant changes were observed from the baseline in the DHI total scale and subscales at 2 weeks and 1, 3, and 6 months in both groups (all $p<0.05$ ). However, the differences between the two groups were not significant (all $p>0.05$ ). Furthermore, we noted significant changes from the baseline in SF-36 scores at 2 weeks in both groups (all $p<0.05$ ), whereas CSF-36 scores were not significantly higher in the SCM group (all $p>0.05$ ) compared with the TM group. No serious AEs were reported in either of the two groups.

Conclusions: No differences in outcomes were detected between the SCM and TM groups in terms of treatment of cervicogenic dizziness. Efficacy trials are required to determine whether the improvement observed for each treatment was causally related to the interventions.
\end{abstract}

Keywords: cervical vertigo, Shi-style cervical mobilizations, traditional massage, randomized controlled clinical trial

\footnotetext{
${ }^{1}$ Institute of Spine Disease, Longhua Hospital, Shanghai University of Traditional Chinese Medicine, Shanghai, China.

${ }^{2}$ Rehabilitation Department, Longhua Hospital, Shanghai University of Traditional Chinese Medicine, Shanghai, China.

${ }^{3}$ Orthopaedics and Traumatology, First Teaching Hospital, Tianjin University of Traditional Chinese Medicine, Tianjin, China.

${ }^{4}$ Rehabilitation Department, Rehabilitation Hospital, Fujian University of Traditional Chinese Medicine, Fuzhou, China.

${ }^{5}$ Tuina Department, Xinjiang Uygur Autonomous Region Hospital of Traditional Chinese Medicine, Urumqi, China.

${ }^{6}$ Orthopaedics and Traumatology, First Affiliated Hospital, Henan University of Traditional Chinese Medicine, Zhengzhou, China.
} 


\section{Introduction}

$\mathbf{C}$ ERVICAL VERTIGO IS A TYPE of dizziness that is provoked by a particular neck posture. ${ }^{1,2}$ It is one of the most common causes of vertigo and affects $\sim 30 \%$ of older people worldwide. ${ }^{3}$ The primary mechanisms underlying cervical vertigo include cervical disorders such as neck articulation, muscle spasms and neck pain, ${ }^{4,5}$ soft tissue injuries of the neck, ${ }^{6}$ cervical degeneration, and conditions associated with decreased vertebrobasilar blood flow including vertebrobasilar dysfunction and subclavian steal. ${ }^{7,8}$ Cervical vertigo severely affects the daily life and employment of affected individuals, and the cost-inefficiency of treatment is an additional burden to patients.

Manual therapies, including manipulation, mobilization, and massage, are predominant methods for treating cervical vertigo. ${ }^{9}$ However, few studies have evaluated the efficacy/ effectiveness of mobilization and massage. One team conducted two randomized control trials (RCTs) evaluating the efficacy of two types of mobilization compared with placebo. They found that mobilization had an immediate and long-term clinically and statistically significant sustained effect in reducing dizziness, cervical pain, and disability caused by cervical dysfunction. ${ }^{10-12}$ With respect to massage, one previous RCT showed that the therapeutic effect of massage might be better than highvelocity manipulation for treating cervical vertigo in younger patients. ${ }^{13}$ However, no previous trials compared the effectiveness of mobilization and massage for treatment of cervical vertigo. A recent systematic review stated that due to the low number of studies, further research is recommended regarding the effect of manual therapies for cervical vertigo. ${ }^{14}$

Shi-style cervical mobilizations (SCM) are a component of Traditional Chinese Medicine. They have a long history and have long been used in clinical practice under the guidance of practitioners trained in classic Chinese materialism and dialectics. The comparative effectiveness and safety of SCM for treatment of cervical spondylotic radiculopathy were objectively evaluated by one RCT when used in long-term clinical practice. ${ }^{15}$ The researchers found that in clinical practice, SCM relieved the neck muscle spasms and eliminated swelling to relieve pain in the cervical region.

The present study aimed to investigate the comparative effectiveness and preliminary safety of SCM compared with traditional massage (TM) in cervical vertigo patients.

\section{Methods}

\section{Study design}

This study was designed as a prospective, multicenter, open-label, randomized, controlled clinical trial with a 1:1 allocation ratio. The study was conducted at the Longhua Hospital, which is affiliated with the Shanghai University of Traditional Chinese Medicine, the First Affiliated Hospital of Henan University of Traditional Chinese Medicine, the Second Affiliated Hospital of Fujian University of Traditional Chinese Medicine, the First Affiliated Hospital of Tianjin University of Traditional Chinese Medicine, and the Xinjiang Uygur Autonomous Region Hospital of Traditional Chinese Medicine.

\section{Participants}

Participants aged 18-60 years with cervical vertigo who had not received any treatment for cervical vertigo within the last 2 weeks and who had visited the outpatient departments of the above-mentioned five hospitals were recruited between June 2012 and March 2014. These participants reported (1) non-rotatory dizziness that was described as an imbalance or unsteadiness and was triggered by neck movements or positions, (2) a stiff or painful neck, or both, and were potentially diagnosed as having cervical vertigo. ${ }^{16}$ For the purposes of diagnosing cervical vertigo, senior clinicians from the Orthopedics or Rehabilitation departments conducted a series of clinical tests, including an examination of the range of motion of the cervical spine, cervical muscle palpations, the DixHallpike test, Doppler cerebrovascular ultrasound, and vertebrae X-ray. These tests were conducted with assistance from senior clinicians from the Otology and Neurology departments.

Patients were excluded from this study if they had undergone treatment in the last 2 weeks. We also excluded pregnant patients; those unable to read Chinese; those with conditions for which manual therapy was contraindicated, such as inflammatory diseases, cancer, or other severe systematic diseases; those with other conditions such as otolithiasis, Meniere disease, and brain disease with dizziness as a symptom; and those who refused to comply with the study protocols.

\section{Randomization}

We used a web-based randomization system managed by an independent third-party clinical research organization (Institute of Basic Research in Clinical Medicine, China Academy of Chinese Medical Science) to randomize the participants. A total of 360 patients were randomized into 2 groups using an equal allocation (1:1) algorithm. After screening, patient information, including name, age, gender, and date of birth, was included in the system. The web-based randomization system showed a serial number for each participant and their corresponding group allocation. Concealed allocation was performed before data collection by a researcher not involved in the screening process or patient treatment.

\section{Treatment protocols}

Because of the characteristics of the mobilization and massage treatments, the patients and therapists could not be blinded. The patients were treated with SCM or TM during six 20-min sessions over 2 weeks, with three sessions per week, and an interval of 1 or 2 days between each session. The therapists determined the treatment schedule and confirmed the next session with the patients during the current session.

Physicians. The physicians who participated in the trial were required to fulfill the following criteria: (1) $\geq 3$ years of experience with manual therapy and (2) participation in study training sessions that provided instruction regarding the trial methods, the interventions tested, and standards for performing clinical trials (International Conference on Harmonization of Technical Requirements for Registration 
of Pharmaceuticals for Human Use- Good Clinical Practice). A total of 41 practitioners in five outpatient units in China participated in this study. A skilled physician taught them SCM and TM. To maximize standardization, all clinicians were provided with on-site training about SCM and TM as well as an instruction manual and a video. Only the skilled physician was qualified to determine whether the trained clinicians could participate in the trial.

\section{Treatments}

SCM group

Soothing tendon step: The therapist kneaded the patient's neck, grasped their back and waist sequentially, and rolled their upper limbs. This was repeated three to six times.

Mobilization step: A low-velocity and small amplitude oscillatory movement without thrust was applied to the neck joint. The therapist lifted the patient's head gently and relaxed their neck by turning their head in the flexion, extension, right $\left(45^{\circ}\right)$, and left $\left(45^{\circ}\right)$ directions. This was repeated three to six times. Then, a pulling force was applied to the neck joint if no discomfort was reported by the patient.

Dredging collateral step: The therapist held the thenar and hypothenar muscles of the hand on the affected side with both hands and gently shook the upper limb up and down in a continuous movement with a small range (small shaking and high frequency). This was repeated three times. Then, the sensitive points of the ears were twisted for $30 \mathrm{sec}$. The last step was to rub the patient's Mingmen (GV 4), Dazhui (GV 14), Naohu (GV 17), and Baihui (GV 20) acupoints for $30 \mathrm{sec}$ each. ${ }^{15}$

Massage group. The massage group was treated according to the National Higher Medical College planning materials regarding "Massage", $(2003)^{17}$ :

Release step: The therapist kneaded the neck, back of the occipital, scapular, and transverse process nodules, and thoracic Jiaji operation via the push method, roll method, and thumb kneading method, focusing on stimulation of the cervical Jiaji, Tianding (LI17), and Tianzong (SI11) acupoints and pain points.

Adjustment step: The therapist applied a pulling force to the neck joint with the patient in a sitting position.

Finishing step: The therapist stimulated both sides of the Fengchi (GB20) and cervical Jianjing (GB21) acupoints and then pushed both sides of the Jianjing (GB21) acupoints in an outward direction using their fingers and palm.

\section{Assessments and follow-up}

The outcomes were assessed before the first treatment as the baseline measurement and then again at 2 weeks, 1 month, and 3 months after the randomization to compare the short-term comparative effectiveness. The outcomes were also assessed at 6 months to compare the intermediateterm comparative effectiveness. We included data from patients who were not able to complete the follow-up interview along with the reason. The participants received instructions from the researchers regarding how to complete the questionnaires. In addition, the patients were asked to report their drug use, including over-the-counter analgesics during the initial 2 weeks after randomization.

\section{Outcome measures}

Primary outcome. The primary outcome was the score on the Dizziness Handicap Inventory (DHI) total scale at 2 weeks after the start of the experiment. This scale was developed to measure the self-perceived level of handicap associated with the symptom of dizziness. ${ }^{18}$ The DHI consists of 25 items with 3 response levels that are categorized into subgroups based on 3 domains: functional, emotional, and physical. The DHI scores were positively correlated with the severity of cervical vertigo. The lower the score, the lesser the degree of disability, which was based on a functional score of 36 points, an emotional score of 36 points, and a body score of 28 points. ${ }^{19}$ The Chinese version of the DHI exhibited optimal reliability and validity, indicating that it could be used to evaluate dizziness. ${ }^{19}$

Secondary outcomes. As secondary outcomes, we used scores on the three DHI subscales, DHI-physical, DHIfunctional, and DHI-emotional at 2 weeks, 1 month, 3 months, and 6 months, as well as scores on the DHI total scale at 1,3 , and 6 months. We also measured the healthrelated quality of life as other secondary outcome using the Chinese version of the Short-Form 36 Health Survey (CSF-36) at 2 weeks. $^{20,21}$ The CSF-36 contains 36 items that represent 8 domains: physical functioning, role-physical, bodily pain, general health, vitality, social functioning, role-emotional, and mental health. ${ }^{21}$ If the participant was confused regarding any items on the questionnaire, the assessor clarified the question. High scores indicated an improved health-related quality of life. The CSF-36 has been found to possess satisfactory validity and reliability but small responsiveness. ${ }^{21}$

Safety. Safety was assessed via spontaneous reporting of adverse events (AEs). We classified serious AEs as those that caused death, were life threatening, or necessitated admission to the hospital. The AEs were actively assessed by the safety evaluators using a specific list at each session during the treatment period. The safety evaluators were blinded to group assignment and recorded the time of occurrence, severity, duration, and outcome of AEs. Also, any side effects of the treatment were recorded at each visit.

Adherence. We asked the participants to avoid receiving any advice or education regarding new treatment strategies and requested that they do not undergo any type of treatment, including drugs, physiotherapy, or manual therapy for cervical vertigo during the treatment period. We monitored whether the participants received any care outside the study during the treatment period by checking the Hospital Information System and questioning the patients at each session.

\section{Quality assurance}

Subcenter quality inspectors ensured that all items on a quality assurance checklist were complete, including informed consent and forms of data storage. To guarantee the quality of the whole trial, we trained five quality inspectors. These inspectors visited each subcenter regularly without 


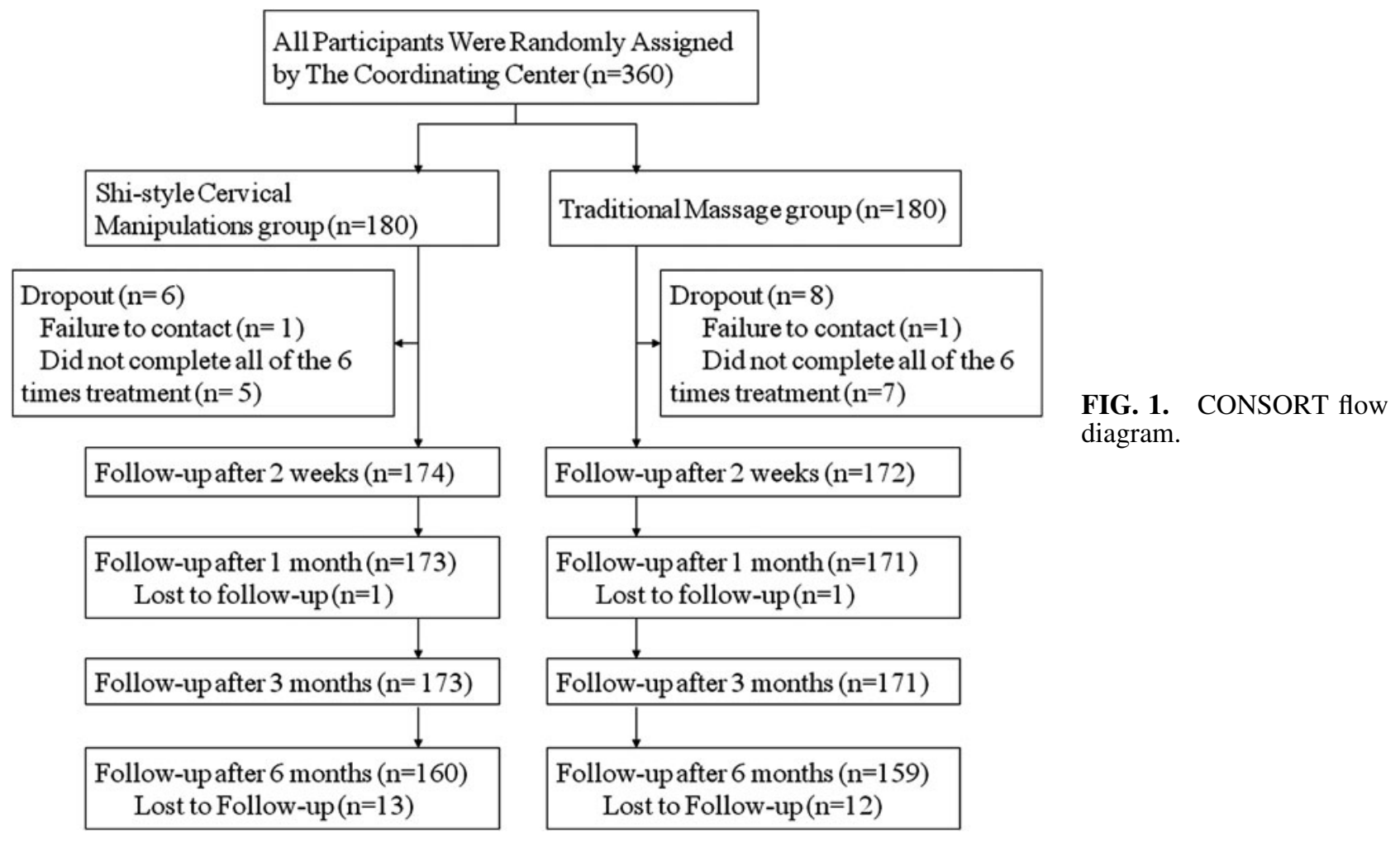

prior notice. To judge the normalization of the trial, all the patients were telephoned and asked about the details of the trial such as the informed consent, the timing of the follow-up assessments, and the quality of the treatments. Any problem occurring in a subcenter was reported in writing by the inspectors. Then, the general center addressed these issues and provided feedback to the other subcenters.

\section{Statistical analyses}

The outcome data were analyzed using Statistical Package for Social Sciences 15.0 software.

The sample size was determined in advance with a 1:1 ratio. The calculation was based on detecting a 12-point between-group differences (a change over $10 \%$ related to clinical change) in the DHI total scale score 2 weeks after treatment, assuming a standard deviation of 17 points from both groups, as reported in other studies. ${ }^{11,22}$ Assuming a dropout rate of $20 \%, \alpha=0.05$, and a power of $90 \%$ with a two-tailed test, we required 180 patients in each group.

All analyses were performed by an independent thirdparty clinical research organization (Institute of Basic Research in Clinical Medicine, China Academy of Chinese Medical Science). Mean differences (mean \pm standard deviation) were used to describe the measurement data, and categorical variables were reported as frequencies. The primary analysis was performed using an independent $t$-test or Mann-Whitney test that compared the groups at each time point in terms of the primary and secondary outcomes. This was carried out using an intention-to-treat (ITT) analysis, which used the full-set analysis (FAS) including data from randomized subjects who underwent at least one treatment and one follow-up assessment. The primary analysis was then repeated using a complete case
Table 1. Baseline Characteristics OF THE PARTICIPANTS

\begin{tabular}{lcc}
\hline Characteristic & $\begin{array}{c}S C M \\
(\mathrm{n}=174)\end{array}$ & $\begin{array}{c}T M \\
(\mathrm{n}=172)\end{array}$ \\
\hline Gender (male/female) & $38 / 136$ & $30 / 142$ \\
Age (years) & $46.3 \pm 11.8$ & $43.7 \pm 12.5$ \\
Disease duration (days) & $56.6 \pm 105.0$ & $68.9 \pm 180.6$ \\
Occupation (active/retired) & $133 / 41$ & $130 / 42$ \\
Treatment experience (yes/no) & $8 / 167$ & $8 / 164$ \\
Physical examination (positive/negative) & \\
Cervical paravertebral & $164 / 111$ & $155 / 17$ \\
$\quad$ tenderness & $155 / 20$ & $151 / 21$ \\
Cervical occipital tenderness & $121 / 54$ & $131 / 41$ \\
Scapular tenderness & & \\
DHI & $39.0 \pm 19.7$ & $37.8 \pm 19.9$ \\
DHI total scale & $14.2 \pm 5.8$ & $14.5 \pm 6.2$ \\
DHI-physical scale & $15.5 \pm 8.5$ & $14.4 \pm 8.4$ \\
DHI-functional scale & $9.2 \pm 7.7$ & $8.9 \pm 7.5$ \\
DHI-emotional scale & & \\
SF-36 & $74.7 \pm 16.8$ & $77.7 \pm 16.9$ \\
Physical functioning & $31.4 \pm 36.4$ & $38.4 \pm 37.6$ \\
Role-physical & $64.1 \pm 19.3$ & $64.9 \pm 17.9$ \\
Bodily pain & $46.1 \pm 17.2$ & $50.1 \pm 16.8$ \\
General health & $56.4 \pm 17.7$ & $59.7 \pm 16.7$ \\
Vitality & $69.0 \pm 19.3$ & $72.9 \pm 18.2$ \\
Social functioning & $47.6 \pm 40.9$ & $53.0 \pm 40.2$ \\
Role-emotional & $62.3 \pm 17.5$ & $64.1 \pm 15.8$ \\
Mental health & & \\
\hline & & \\
Datal & &
\end{tabular}

Data are expressed as mean \pm standard deviation.

DHI, Dizziness Handicap Inventory; SCM, Shi-style cervical manipulations; TM, traditional massage. 
analysis to gain insight into the effects of missing data. The complete case analysis included all patients who completed the treatments and follow-up interviews. As the secondary analysis, a one-way repeated-measures analysis of variance was adopted to investigate the changes within each group after the intervention in terms of DHI total and subscale scores while the Wilcoxon rank-sum test was used to investigate the changes in CSF-36 data at 2 weeks.

\section{Results}

Recruitment

Between February 2012 and May 2014, 360 patients were assessed for eligibility. Figure 1 shows a schematic illustration of the patient selection process. All the participants provided written informed consent, and one withdrew their consent after learning about the treatment.

Table 2. Dizziness Handicap Inventory: Full-Set Analysis

\begin{tabular}{|c|c|c|c|c|}
\hline & $S C M(\mathrm{n}=174)$ & $T M(\mathrm{n}=172)$ & $\begin{array}{l}\text { Mean difference } \\
\text { between groups }\end{array}$ & $\mathrm{p}$ \\
\hline \multicolumn{5}{|l|}{ DHI total scale } \\
\hline Baseline & $39.0 \pm 19.7$ & $37.8 \pm 19.9$ & & \\
\hline 2 Weeks & $21.8 \pm 16.1$ & $22.9 \pm 15.9$ & $-1.1 \pm 22.7[-4.5$ to 2.2$]$ & 0.507 \\
\hline Change & $17.2 \pm 36.4[11.7$ to 22.7$]$ & $14.9 \pm 36.2[9.4$ to 20.3$]$ & & \\
\hline 1 Month & $18.6 \pm 15.4$ & $19.9 \pm 16.6$ & $-1.3 \pm 22.7[-4.7$ to 2.1$]$ & 0.459 \\
\hline Change & $20.4 \pm 35.8[15.0$ to 25.8$]$ & $17.9 \pm 36.5[12.4$ to 23.5$]$ & & \\
\hline 3 Months & $16.3 \pm 16.0$ & $18.1 \pm 17.0$ & $-1.8 \pm 24.0[-5.4$ to 1.8$]$ & 0.316 \\
\hline Change & $22.7 \pm 36.5[17.2$ to 28.2$]$ & $19.7 \pm 37.9[14.0$ to 25.3$]$ & & \\
\hline 6 Months & $15.5 \pm 17.0$ & $15.3 \pm 15.6$ & $0.2 \pm 23.9[-3.4$ to 3.8$]$ & 0.920 \\
\hline Change & $23.5 \pm 37.9[17.8$ to 29.2$]$ & $22.5 \pm 36.5[17.0$ to 28.0$]$ & & \\
\hline $\begin{array}{l}p \text {-Value for one-way } \\
\text { repeated-measures ANOVA }\end{array}$ & $<0.001$ & $<0.001$ & & \\
\hline \multicolumn{5}{|l|}{ DHI-physical scale } \\
\hline Baseline & $14.2 \pm 5.8$ & $14.5 \pm 6.2$ & & \\
\hline 2 Weeks & $8.1 \pm 4.9$ & $9.1 \pm 5.3$ & $-1.0 \pm 7.3[-2.1$ to 0.1$]$ & 0.066 \\
\hline Change & $6.1 \pm 7.0[4.5$ to 7.7$]$ & $5.4 \pm 11.6[3.6$ to 7.1$]$ & & \\
\hline 1 Month & $7.3 \pm 4.9$ & $7.9 \pm 5.6$ & $-0.6 \pm 7.3[-1.7$ to 0.5$]$ & 0.270 \\
\hline Change & $6.9 \pm 7.4[5.8$ to 8.0$]$ & $6.6 \pm 11.9[4.8$ to 8.3$]$ & & \\
\hline 3 Months & $6.3 \pm 4.0$ & $7.1 \pm 6.0$ & $-0.8 \pm 8.0[-2.0$ to 0.4$]$ & 0.178 \\
\hline Change & $7.9 \pm 11.0[6.2$ to 9.5$]$ & $7.3 \pm 12.3[5.5$ to 9.2$]$ & & \\
\hline 6 Months & $3.5 \pm 5.5$ & $3.4 \pm 4.6$ & $0.2 \pm 21.3[-3.4$ to 3.8$]$ & 0.970 \\
\hline Change & $8.1 \pm 11.7[6.3$ to 9.8$]$ & $8.4 \pm 12.1[6.5$ to 10.2$]$ & & \\
\hline $\begin{array}{l}p \text {-Value for one-way } \\
\text { repeated-measures ANOVA }\end{array}$ & $<0.001$ & $<0001$ & & \\
\hline \multicolumn{5}{|l|}{ DHI-functional scale } \\
\hline Baseline & $15.5 \pm 8.5$ & $14.4 \pm 8.4$ & & \\
\hline 2 Weeks & $8.5 \pm 7.4$ & $8.7 \pm 6.6$ & $-0.2 \pm 10.0[-1.7$ to 1.3$]$ & 0.837 \\
\hline Change & $7.0 \pm 16.2[4.6$ to 9.5$]$ & $5.8 \pm 15.3[3.5$ to 8.1$]$ & & \\
\hline 1 Month & $7.0 \pm 7.0$ & $7.4 \pm 6.7$ & $-1.3 \pm 3.3[-1.8$ to 1.1$]$ & 0.270 \\
\hline Change & $8.5 \pm 15.9[6.1$ to 10.9$]$ & $7.0 \pm 11.9[5.2$ to 9.9$]$ & & \\
\hline 3 Months & $6.3 \pm 7.2$ & $6.8 \pm 6.9$ & $-0.6 \pm 10.0[-2.1$ to 0.9$]$ & 0.449 \\
\hline Change & $9.2 \pm 16.1[6.9$ to 11.7$]$ & $7.6 \pm 15.6[5.2$ to 9.9$]$ & & \\
\hline 6 Months & $5.9 \pm 7.4$ & $5.8 \pm 6.6$ & $0.1 \pm 10.0[-1.4$ to 1.7$]$ & 0.858 \\
\hline Change & $9.6 \pm 16.4[7.2$ to 12.1$]$ & $8.7 \pm 15.4[6.3$ to 11.0$]$ & & \\
\hline $\begin{array}{l}p \text {-Value for one-way } \\
\text { repeated-measures ANOVA }\end{array}$ & $<0.001$ & $<0.001$ & & \\
\hline \multicolumn{5}{|l|}{ DHI-emotional scale } \\
\hline Baseline & $9.3 \pm 7.7$ & $8.9 \pm 7.5$ & & \\
\hline 2 Weeks & $5.2 \pm 5.7$ & $5.1 \pm 5.6$ & $0.0 \pm 8.0[-1.2$ to 1.2$]$ & 0.967 \\
\hline Change & $4.1 \pm 13.6[2.0$ to 6.1$]$ & $3.8 \pm 13.3[1.8$ to 5.8$]$ & & \\
\hline 1 Month & $4.3 \pm 5.3$ & $4.6 \pm 5.7$ & $-0.3 \pm 8.0[-1.5$ to 0.9$]$ & 0.634 \\
\hline Change & $5.0 \pm 13.3[3.0$ to 7.0$]$ & $4.3 \pm 13.5[2.3$ to 6.4$]$ & & \\
\hline 3 Months & $3.7 \pm 5.4$ & $4.1 \pm 5.5$ & $-0.4 \pm 8.0[-1.6$ to 0.8$]$ & 0.478 \\
\hline Change & $5.5 \pm 13.5[3.5$ to 7.6$]$ & $4.8 \pm 13.3[2.8$ to 6.8$]$ & & \\
\hline 6 Months & $3.5 \pm 5.5$ & $3.4 \pm 4.6$ & $0.0 \pm 7.3[-1.1$ to 1.1$]$ & 0.972 \\
\hline Change & $5.8 \pm 13.6[3.8$ to 7.9$]$ & $5.5 \pm 12.7[3.6$ to 7.4$]$ & & \\
\hline $\begin{array}{l}p \text {-Value for one-way } \\
\text { repeated-measures ANOVA }\end{array}$ & $<0.001$ & $<0.001$ & & \\
\hline
\end{tabular}

Baseline and final data are expressed as mean \pm standard deviation; change data and mean difference between groups are expressed as mean \pm standard deviation [95\% confidence interval].

ANOVA, analysis of variance; DHI, Dizziness Handicap Inventory; SCM, Shi-style cervical manipulations; TM, traditional massage. 
Table 3. Dizziness Handicap Inventory: Complete Case Analysis

\begin{tabular}{|c|c|c|c|c|}
\hline & $S C M(\mathrm{n}=160)$ & $T M(\mathrm{n}=159)$ & $\begin{array}{l}\text { Mean difference } \\
\text { between groups }\end{array}$ & $\mathrm{p}$ \\
\hline \multicolumn{5}{|l|}{ DHI total scale } \\
\hline Baseline & $39.7 \pm 19.5$ & $38.5 \pm 20.4$ & & \\
\hline 2 Weeks & $22.2 \pm 16.4$ & $23.0 \pm 16.3$ & $-0.8 \pm 24.0[-4.4$ to 2.9$]$ & 0.655 \\
\hline Change & $17.5 \pm 36.5[11.8$ to 23.1$]$ & $15.4 \pm 37.1[9.6$ to 21.3$]$ & & \\
\hline 1 Month & $19.1 \pm 15.5$ & $20.0 \pm 17.0$ & $-0.9 \pm 23.3[-4.4$ to 2.7$]$ & 0.363 \\
\hline Change & $20.5 \pm 35.2[15.0$ to 26.1$]$ & $18.5 \pm 38.4[12.5$ to 24.4$]$ & & \\
\hline 3 Months & $16.3 \pm 16.1$ & $18.0 \pm 17.1$ & $-1.8 \pm 24.0[-5.4$ to 1.9$]$ & 0.341 \\
\hline Change & $23.4 \pm 35.8[17.8$ to 29.1$]$ & $20.4 \pm 37.7[14.5$ to 26.4$]$ & & \\
\hline 6 Months & $15.3 \pm 7.0$ & $15.3 \pm 15.6$ & $0.0 \pm 24.0[-3.6$ to 3.6$]$ & 0.993 \\
\hline Change & $24.4 \pm 37.1[18.6$ to 30.1$]$ & $23.2 \pm 37.1[17.4$ to 28.9$]$ & & \\
\hline $\begin{array}{l}p \text {-Value for one-way } \\
\text { repeated-measures ANOVA }\end{array}$ & $<0.001$ & $<0.001$ & & \\
\hline \multicolumn{5}{|l|}{ DHI-physical scale } \\
\hline Baseline & $14.4 \pm 5.6$ & $14.9 \pm 6.3$ & & \\
\hline 2 Weeks & $8.3 \pm 4.9$ & $9.1 \pm 5.4$ & $-0.8 \pm 7.3[-1.9$ to 0.4$]$ & 0.182 \\
\hline Change & $6.1 \pm 10.8[4.4$ to 7.8$]$ & $5.5 \pm 11.5[3.7$ to 7.3$]$ & & \\
\hline 1 Month & $7.5 \pm 4.8$ & $7.9 \pm 5.6$ & $-0.3 \pm 8.0[-1.5$ to 0.8$]$ & 0.565 \\
\hline Change & $6.9 \pm 10.8[5.2$ to 8.5$]$ & $6.7 \pm 12.1[4.8$ to 8.6$]$ & & \\
\hline 3 Months & $6.3 \pm 5.0$ & $7.1 \pm 5.9$ & $-0.8 \pm 8.0[-2.0$ to 0.4$]$ & 0.197 \\
\hline Change & $8.1 \pm 10.8[6.4$ to 9.8$]$ & $7.5 \pm 12.8[5.5$ to 9.4$]$ & & \\
\hline 6 Months & $6.1 \pm 5.6$ & $6.1 \pm 5.6$ & $0.0 \pm 8.6[-1.3$ to 1.2$]$ & 0.952 \\
\hline Change & $8.4 \pm 11.5[6.6$ to 10.1$]$ & $8.5 \pm 12.1[6.6$ to 10.4$]$ & & \\
\hline $\begin{array}{l}p \text {-Value for one-way } \\
\text { repeated-measures ANOVA }\end{array}$ & $<0.001$ & $<0001$ & & \\
\hline \multicolumn{5}{|l|}{ DHI-functional scale } \\
\hline Baseline & $15.9 \pm 8.4$ & $14.7 \pm 8.6$ & & \\
\hline 2 Weeks & $8.7 \pm 7.5$ & $8.7 \pm 6.8$ & $-0.1 \pm 10.0[-1.6$ to 1.5$]$ & 0.993 \\
\hline Change & $7.2 \pm 16.0[4.7$ to 9.7$]$ & $6.0 \pm 16.0[3.5$ to 8.4$]$ & & \\
\hline 1 Month & $7.3 \pm 7.1$ & $7.4 \pm 6.8$ & $-0.2 \pm 10.0[-1.7$ to 1.4$]$ & 0.826 \\
\hline Change & $8.6 \pm 15.3[6.2$ to 11.1$]$ & $7.3 \pm 16.0[4.8$ to 9.7$]$ & & \\
\hline 3 Months & $6.2 \pm 7.2$ & $6.8 \pm 7.0$ & $-0.6 \pm 10.0[-2.1$ to 1.0$]$ & 0.476 \\
\hline Change & $9.7 \pm 16[7.2$ to 12.1$]$ & $7.9 \pm 16.0[5.4$ to 10.4$]$ & & \\
\hline 6 Months & $5.8 \pm 7.3$ & $5.8 \pm 6.6$ & $0.1 \pm 10.6[-1.5$ to 1.6$]$ & 0.992 \\
\hline Change & $10.1 \pm 16.0[7.6$ to 12.5$]$ & $8.9 \pm 15.3[6.5$ to 11.4$]$ & & \\
\hline $\begin{array}{l}p \text {-Value for one-way } \\
\text { repeated-measures ANOVA }\end{array}$ & $<0.001$ & -0001 & & \\
\hline \multicolumn{5}{|l|}{ DHI-emotional scale } \\
\hline Baseline & $9.4 \pm 7.8$ & $9.2 \pm 7.7$ & & \\
\hline 2 Weeks & $5.2 \pm 5.8$ & $5.2 \pm 5.7$ & $0.0 \pm 8.7[-1.3$ to 1.3$]$ & 0.978 \\
\hline Change & $4.2 \pm 14.0[2.0$ to 6.3$]$ & $4.0 \pm 14.0[1.8$ to 6.1$]$ & & \\
\hline 1 Month & $4.4 \pm 5.4$ & $4.7 \pm 5.9$ & $-0.4 \pm 8.0[-1.6$ to 0.8$]$ & 0.576 \\
\hline Change & $5.0 \pm 13.4[2.9$ to 7.1$]$ & $4.5 \pm 14.0[2.3$ to 6.6$]$ & & \\
\hline 3 Months & $3.7 \pm 5.4$ & $4.1 \pm 5.5$ & $-0.4 \pm 8.0[-1.6$ to 0.8$]$ & 0.500 \\
\hline Change & $5.7 \pm 13.4[3.6$ to 7.8$]$ & $5.1 \pm 14.0[2.9$ to 7.2$]$ & & \\
\hline 6 Months & $3.4 \pm 5.4$ & $3.4 \pm 4.6$ & $-0.0 \pm 7.4[-1.1$ to 1.1$]$ & 0.970 \\
\hline Change & $6.0 \pm 14.0[3.8$ to 8.1$]$ & $5.7 \pm 12.8[3.7$ to 7.8$]$ & & \\
\hline $\begin{array}{l}p \text {-Value for one-way } \\
\text { repeated-measures ANOVA }\end{array}$ & $<0.001$ & $<0.001$ & & \\
\hline
\end{tabular}

Baseline and final data are expressed as mean \pm standard deviation; change data and mean difference between groups are expressed as mean \pm standard deviation [95\% confidence interval].

ANOVA, analysis of variance; DHI, Dizziness Handicap Inventory; SCM, Shi-style cervical manipulations; TM, traditional massage.

\section{Patient characteristics}

Table 1 presents the characteristics of the patients. Over $80 \%$ of the participants were women. The mean duration of cervical vertigo was 56.6 \pm 105.0 days in the SCM group and $68.9 \pm 180.6$ days in the TM group. Approximately $95 \%$ of the patients had not previously received a normative treatment.

\section{Treatment adherence}

In both groups, the majority of the patients underwent six sessions in 2 weeks. One patient in each of the SCM and TM groups underwent only one session because of failure to maintain contact, whereas three patients in the SCM group and two patients in the TM group received only one session due to a lack of availability. Two patients in the SCM group 
and five patients in the TM group declined further treatment because of minimal effectiveness. None of the patients was treated with drugs or received other types of treatment for cervical vertigo during the treatment period.

\section{Follow-up}

A total of $174(96.67 \%)$ participants in the SCM group and $172(95.56 \%)$ patients in the TM group completed at least one treatment session and at least one follow-up assessment, whereas $160(88.89 \%)$ patients in the SCM group and $159(88.33 \%)$ patients in the TM group completed all the follow-up assessments. The proportion of patients who did not complete the trial was similar between the two groups. The loss to follow-up in the SCM group was attributed to a lack of availability regarding the interviews. This was the case for 18 patients in the SCM group and 17 patients in the TM group.

\section{Primary outcome}

$\mathrm{DHI}$ total scale. Table 2 presents the full-set ITT analysis for the DHI total scale. Statistically and clinically significant improvement from baseline was detected at each follow-up time point in both groups (mean improvement $>15, p<0.001$ ). Conversely, there were no statistically and clinically significant differences between groups at 2 weeks (mean difference $=$
$-0.8, p=0.507), 1$ months $(-0.9, p=0.459), 3$ months $(-1.8$, $p=0.316)$, and 6 months $(0.0, p=0.920)$. Similar results were observed in the complete case analysis (Table 3 ).

\section{Secondary outcomes}

DHI subscales. Table 2 also presents the DHI subscales based on the full-set analysis. Statistically significant changes from baseline were detected in the DHI-physical, DHIfunctional, and DHI-emotional scales throughout the whole follow-up in both groups $(p<0.001)$. However, differences between groups were small for the three subscales at all follow-ups $(p>0.05)$. Similar results were observed in the complete case analysis (Table 3 ).

CSF-36. Table 4 presents the CSF-36 based on the full-set analysis. In terms of the FAS data, we detected significant changes from baseline in CSF-36 at 2 weeks in both groups (all $p<0.05$ ). However, the SF-36 was not significantly higher in the SCM group (all $p>0.05$ ) than in the TM group.

Safety. No serious AEs were reported. Two patients in the SCM group and one patient in the TM group reported nausea. This expected, nonserious AEs was self-limited, and no permanent injuries occurred. The affected patients refused to continue future treatments.

Table 4. Chinese Version of the Short-Form 36 Health Survey: Full-Set Analysis

\begin{tabular}{|c|c|c|c|c|}
\hline & $S C M(\mathrm{n}=174)$ & $T M(\mathrm{n}=172)$ & Mean difference between groups & $\mathrm{p}$ \\
\hline \multicolumn{5}{|l|}{ CSF-36 } \\
\hline Baseline & $56.5 \pm 15.9$ & $60.1 \pm 16.2$ & & \\
\hline 2 Weeks & $63.9 \pm 15.9$ & $66.4 \pm 15.6$ & $-2.5 \pm 22.7[5.9$ to 0.8$]$ & 0.131 \\
\hline \multicolumn{5}{|c|}{ Physical functioning } \\
\hline Baseline & $74.7 \pm 16.8$ & $77.7 \pm 16.9$ & & \\
\hline 2 Weeks & $81.5 \pm 14.2$ & $82.8 \pm 13.9$ & $-1.3 \pm 20.7[-4.4$ to 1.6$]$ & 0.376 \\
\hline \multicolumn{5}{|c|}{ Role-physical } \\
\hline Baseline & $31.4 \pm 36.4$ & $38.4 \pm 37.6$ & & \\
\hline 2 Weeks & $49.0 \pm 42.3$ & $53.5 \pm 40.7$ & $-4.5 \pm 58.8[-13.3$ to 4.3$]$ & 0.334 \\
\hline \multicolumn{5}{|l|}{ Bodily pain } \\
\hline Baseline & $64.1 \pm 19.3$ & $64.9 \pm 17.9$ & & \\
\hline 2 Weeks & $72.1 \pm 16.9$ & $72.3 \pm 16.0$ & $-0.2 \pm 23.3[-3.7$ to 3.3$]$ & 0.799 \\
\hline \multicolumn{5}{|c|}{ General health } \\
\hline Baseline & $46.1 \pm 17.2$ & $50.1 \pm 16.8$ & & \\
\hline 2 Weeks & $50.5 \pm 17.1$ & $52.8 \pm 16.3$ & $-2.2 \pm 24.0[-5.8$ to 1.3$]$ & 0.289 \\
\hline \multicolumn{5}{|l|}{ Vitality } \\
\hline Baseline & $56.4 \pm 17.7$ & $59.7 \pm 16.7$ & & \\
\hline 2 Weeks & $60.4 \pm 16.2$ & $62.8 \pm 15.4$ & $-2.5 \pm 22.0[-5.8$ to 0.9$]$ & 0.144 \\
\hline \multicolumn{5}{|c|}{ Social functioning } \\
\hline Baseline & $69.0 \pm 19.3$ & $72.9 \pm 18.2$ & & \\
\hline 2 Weeks & $73.4 \pm 18.5$ & $76.7 \pm 16.4$ & $-3.3 \pm 24.7[-7.0$ to -0.4$]$ & 0.164 \\
\hline \multicolumn{5}{|c|}{ Role-emotional } \\
\hline Baseline & $47.6 \pm 40.9$ & $53.0 \pm 40.2$ & & \\
\hline 2 Weeks & $58.9 \pm 40.3$ & $64.7 \pm 39.3$ & $-5.9 \pm 56.1[-14.3$ to 2.8$]$ & 0.149 \\
\hline \multicolumn{5}{|c|}{ Mental health } \\
\hline Baseline & $62.3 \pm 17.5$ & $64.1 \pm 15.8$ & & \\
\hline 2 Weeks & $65.6 \pm 16.7$ & $65.9 \pm 15.3$ & $0.3 \pm 26.7[-3.7$ to 3.1$]$ & 0.900 \\
\hline
\end{tabular}

Baseline and final data are expressed as mean \pm standard deviation; the mean difference between groups is expressed as mean \pm standard deviation [95\% confidence interval].

CSF-36, Chinese version of the Short-Form 36 Health Survey; SCM, Shi-style cervical manipulations; TM, traditional massage. 


\section{Discussion}

To the best of our knowledge, this is the first randomized controlled trial investigating the comparative effectiveness of the mobilization compared with the massage for the treatment of cervical vertigo. No evidence for superiority of mobilization or massage was found in the DHI and SF-36 outcomes in either the short term or the intermediate term. However, it should be pointed out that the narrow confidence intervals in Table 2 for the DHI total score, which are much narrower than a minimal clinically important difference between groups, suggest that the effects of these two interventions on cervicogenic vertigo may be quite similar.

The advantages of this study included a large sample of patients, a randomized controlled design, and high followup rates. The compliance rates for the mobilization and massage treatments were higher than those reported in other trials. The trial had an ideal quality assurance plan to ensure the authenticity and reliability of the data. Each of the branch centers had a trained quality inspector to ensure the authenticity of each patient and guarantee the quality of the data. Nevertheless, this study had some limitations. Similar to other studies on manual therapies, blinding was impossible. The outcome measures, such as the DHI and SF-36, rely heavily on self-reporting, and thus, their positive outcomes are likely to be overestimated. Moreover, we could not discern whether the treatments were similarly effective or not effective because the efficacy of neither is known. Hence, there is no evidence to suggest that the improvement in each group was causally related to the treatment rather than to natural history or nonspecific effects. In addition, outside care was not monitored during the follow-up period so any associated effects are unknown.

\section{Conclusions}

We found no differences between the effects of mobilization and massage as treatments for cervical dizziness. Further study regarding the efficacy of these treatments is required to determine whether the improvement associated with both treatments was causally related to the interventions.

\section{Author Disclosure Statement}

The authors declare no competing interests.

\section{Authors' Contributions}

Q.S. and Y.-J.W. conceptualized the study. X.-J.C. and M.Y. designed the trial variables and obtained the funding. Q.S. and Y.-J.W. supervised the study. M.Y., Z.-Y.T., X.-J.C., Y.-L.S., X.-L.Y., P.W., W.-H.Z., R.-C.Z., H.-Y.L., Z.-J.H., W.-M.W., W.-P.Q., J.L., and Y.G. collected the data. M.Y. and Z.-Y.T. carried out the statistical analysis. M.Y. drafted the article, which was revised by Z.-Y.T. and Y.-J.W.

\section{Disclaimer}

The funding agencies did not play any role in the study design and collection, analysis, and interpretation of data, writing of the report, or in the decision to submit the article for publication.

\section{Patient Consent}

Obtained.

\section{Ethics Approval}

The authors assert that all procedures contributing to this work comply with the ethical standards of the relevant national and institutional committees on human experimentation and with the Declaration of Helsinki 1975, as revised in 2008. The study was approved by the Research Ethics Committee at the Longhua Hospital, Shanghai University of Traditional Chinese Medicine (No. 2011LCSY032). No amendments were made after the trial started.

\section{Data Sharing Statement}

No additional data are available.

\section{Funding Information}

This is a special project of the National Natural Science Foundation of China (81704096, 81603635, 81330085), the Program for Innovative Research Team of Ministry of Science and Technology of China (2015RA4002), the Program for Innovative Research Team of Ministry of Education of China (IRT1270), Municipal Science and Technology Commission of Shanghai-TCM Key Project (16401970100), Shanghai TCM Medical Center of Chronic Disease (2017ZZ01010), National Thirteenth Five-Year Science and Technology Major Special Project for New Drug Innovation and Development (2017ZX09304001), and Essential Drug Research and Development from Ministry of Science and Technology (2019ZX09201004-003-032).

\section{References}

1. Hain TC. Cervicogenic causes of vertigo. Curr Opin Neurol 2015;28:69-73.

2. Yahia A, Ghroubi S, Jribi S, et al. Chronic neck pain and vertigo: Is a true balance disorder present? Ann Phys Rehabil Med 2009;52:556-567.

3. Colledge N, Lewis S, Mead G, et al. Magnetic resonance brain imaging in people with dizziness: A comparison with non-dizzy people. J Neurol Neurosurg Psychiatry 2002;72: 587-589.

4. Morinaka S. Musculoskeletal diseases as a causal factor of cervical vertigo. Auris Nasus Larynx 2009;36:649-654.

5. Schweigart G, Chien RD, Mergner T. Neck proprioception compensates for age-related deterioration of vestibular selfmotion perception. Exp Brain Res 2002;147:89-97.

6. Endo K, Ichimaru K, Komagata M, Yamamoto K. Cervical vertigo and dizziness after whiplash injury. Eur Spine J 2006;15:886-890.

7. Peng B. Cervical vertigo: Historical reviews and advances. World Neurosurg 2018;109:347-350.

8. Wolf PA, D'Agostino RB, O'Neal MA, et al. Secular trends in stroke incidence and mortality. The Framingham Study. Stroke 1992;23:1551-1555.

9. Lu DC, Zador Z, Mummaneni PV, et al. Rotational vertebral artery occlusion-series of 9 cases. Neurosurgery 2010; 67:1066-1072.

10. Reid SA, Rivett DA, Katekar MG, et al. Sustained natural apophyseal glides (SNAGs) are an effective treatment for cervicogenic dizziness. Man Ther 2008;13:357-366. 
11. Reid SA, Callister R, Snodgrass SJ, et al. Manual therapy for cervicogenic dizziness: Long-term outcomes of a randomised trial. Man Ther 2015;20:148-156.

12. Reid SA, Callister R, Katekar MG, et al. Effects of cervical spine manual therapy on range of motion, head repositioning, and balance in participants with cervicogenic dizziness: A randomized controlled trial. Arch Phys Med Rehabil 2014; 95:1603-1612.

13. Kang F, Wang QC, Ye YG. A randomized controlled trial of rotatory reduction manipulation and acupoint massage in the treatment of younger cervical vertigo. Zhongguo Gu Shang 2008;21:270-272.

14. Yaseen K, Hendrick P, Ismail A, et al. The effectiveness of manual therapy in treating cervicogenic dizziness: A systematic review. J Phys Ther Sci 2018;30:96-102.

15. Cui XJ, Yao M, Ye XL, et al. Shi-style cervical manipulations for cervical radiculopathy: A multicenter randomizedcontrolled clinical trial. Medicine (Baltimore) 2017;96: e7276.

16. Li Y, Peng B. Pathogenesis, diagnosis, and treatment of cervical vertigo. Pain Physician 2015;18:E583-E595.

17. Yan JT, Wang DQ, Fang M, et al. Massage. Beijing, China: China Press of Traditional Chinese Medicine, 2003:129-135.

18. Jacobson GP, Newman CW. The development of the Dizziness Handicap Inventory. Arch Otolaryngol Head Neck Surg 1990;116:424-427.
19. Zhang Y, Liu B, Wang YZ, et al. Analysis of reliability and validity of the Chinese Version of Dizziness Handicap Inventory (DHI). Zhonghua Er Bi Yan Hou Tou Jing Wai Ke Za Zhi 2015;50:738-743.

20. Wu YF, Xie GQ, Li Y, et al. The development and Assessment on the general quality of life intrument for Chinese people. Zhonghua Liu Xing Bing Xue Za Zhi 2005;26: 751-756.

21. Yang Z, Li W, Tu X, et al. Validation and psychometric properties of Chinese version of SF-36 in patients with hypertension, coronary heart diseases, chronic gastritis and peptic ulcer. Int J Clin Pract 2012;66:991-998.

22. Treleaven J. Dizziness Handicap Inventory (DHI). Aust J Physiother 2006;52:67.

Address correspondence to:

Yong-jun Wang, PhD

Institute of Spine Disease

Longhua Hospital

Shanghai University of Traditional Chinese Medicine 725 South Wanping Road Shanghai 200032

China

E-mail: wangyongjuntcm@sina.com 